\title{
Cone states of tri-hydrogen isotopomers and criterion for the geometric phase effect
}

\author{
A.J.C. Varandas *, L.P. Viegas \\ Departamento de Química, Universidade de Coimbra 3004-535 Coimbra, Portugal \\ Received 23 July 2002; in final form 28 October 2002
}

\begin{abstract}
We report calculations of the vibrational spectrum of $\mu \mathrm{H}_{2} / \mathrm{HD} / \mathrm{DT}$ as well as $\mathrm{D}_{3}$ in their first-excited electronic state, both with the inclusion of the geometric phase effect and without including it. The results show that, especially for $\mu \mathrm{HD}$ and $\mu \mathrm{DT}$, it plays a minor role for the first 30 vibrational levels of such systems. This can be rationalized from the tilting of the $\mathrm{C}_{3}$-axis due to mass effects. A simple phenomenological criterion for the relative role of the GP effect in tri-hydrogen isotopomers has been suggested.
\end{abstract}

(c) 2002 Elsevier Science B.V. All rights reserved.

\section{Introduction}

Tri-hydrogen and its isotopic variants have long been studied, both experimentally and theoretically. In particular, the simplest reaction in nature $\mathrm{H}+\mathrm{H}_{2} \rightarrow \mathrm{H}_{2}+\mathrm{H}$ has been investigated at full quantum state-to-state level both theoretically and experimentally. Regarding the isotopomers, the $\mathrm{D}+\mathrm{H}_{2}$ and $\mathrm{H}+\mathrm{D}_{2}$ exchange reactions have received the most attention [1,2]. However, the first experimental and theoretical study of the reaction $\mathrm{H}+\mathrm{HD} \rightarrow \mathrm{H}_{2}+\mathrm{D}$ has been published only recently [3] at $E=0.5 \mathrm{eV}$; for a quantum study of the transition state dynamics, see [4]. A striking result from the study of Harich et al. [3] is that

\footnotetext{
${ }^{*}$ Corresponding author. Fax: +351-39-827-703. das).
}

'precluding a small influence of the so-called geometric phase (GP) effect or errors in the potential energy surface, it does not appear necessary to include their effects to achieve a very good model of the dynamics'.

The GP effect is a consequence of the conical intersection present between the ground- and firstexcited surfaces. As pointed out by Mead and Truhlar [5] following pioneering work by LonguetHiggins [6,7] and Berry [8], the GP effect should be included whenever a single-surface Born-Oppenheimer treatment is carried out for such systems. Note that the intersection in the tri-hydrogen potential energy surface occurs along the line of equilateral triangular geometries (i.e., the $\mathrm{C}_{3}$-axis), and has its minimum energy value about $2.7 \mathrm{eV}$ above the ground surface limit for atom-diatom dissociation. Wu and Kuppermann [1] observed from dynamics calculations on the $\mathrm{H}+\mathrm{D}_{2}$ reaction that the GP effect may have a strong influence 
even for energies far below the conical intersection. This result is supported by the experimental studies of Zare and co-workers [9], which indicate that calculations including the GP effect give better agreement with their experimental results than calculations not including it (NGP). However, from more recent experiments, Welge and coworkers [2, and references therein] concluded oppositely. Such a conclusion was supported theoretically by Kendrick [10, and references therein] who has shown that GP would have insignificant effect on the dynamics of the $\mathrm{H}+\mathrm{D}_{2}$ system at a total energy of $1.881 \mathrm{eV}$ for values of total angular momentum up to $J=5$. Most recently, Kuppermann and $\mathrm{Wu}$ [11] revisited this problem, and also found that up to $J=5$ the GP effect on differential cross sections is negligibly small. However, as the value of $J$ is increased, differences due the GP effect turned out to be important for the potential energy surface used in their calculations. They have then concluded that calculations that do not include such an effect are conceptually incorrect and, in the case of $\mathrm{H}+\mathrm{D}_{2}$, numerically inaccurate.

The GP effect is also known to play a significant role in molecular spectra [12-17]. Moreover, it has been shown from ab initio calculations on $\mathrm{LiNaK}$ that the GP effect is present even when the system has no symmetry [18]. Thus, it should be taken into account when studying the (reactive or nonreactive) nuclear dynamics of such systems using a single-BO surface. Since vibrational calculations are easier to perform than reactive scattering ones, and because the GP effect should manifest on both as a topological effect, we may choose the former to estimate its importance. Furthermore, one expects the GP effect to manifest more prominently on the upper adiabatic sheet as this always lies above the crossing seam. Thus, if one finds that such an effect does not play a role on the vibrational cone states, it is reasonable to conclude that it will not play a role either for the reaction dynamics on the bottom adiabatic sheet.

Two alternative schemes have been suggested to treat the GP effect. One consists of multiplying the real double-valued electronic wave functions by a complex phase-factor which changes sign on encircling the conical intersection. This makes the resulting complex electronic wave function to be single-valued [19, and references therein], and leads to the vector potential of Mead and Truhlar $[5,20]$. The other method, particularly advantageous for $\mathrm{X}_{3}$-type systems, consists of incorporating the complex phase factor into the nuclear wave function such as to make the total electronuclear wave function single-valued [12,16,17,21]. However, a complication arises when studying the isotopomers of $\mathrm{X}_{3}$, due to the mass scaling involved in defining the hyperspherical coordinates. To cope with this problem, Kuppermann and $\mathrm{Wu}$ [22] employed a mass-scaled Jacobi-vectors formula, while we suggested both a split-basis technique [23] and a coordinate-transformation approach [24].

In this work, we treat the GP effect by solving a generalized Born-Oppenheimer (GBO) equation proposed by Varandas and $\mathrm{Xu}$ [25] following the work of Baer and Englman [26,27]. Such an equation is strictly valid close to the seam (see also [28]), although generality may be conveyed by invoking the well known $[6,7,17,29]$ fact that such regions have a dominant role in the nuclear dynamics even when energetics allows to sample areas of configurational space far away from the seam. It uses the result [25] that the GP angle $A(\mathbf{R})$ is, up to a constant, identical to the mixing angle $\gamma(\mathbf{R})$ of the orthogonal transformation that diagonalizes the potential matrix in the coupled two-states problem. [Note that the adiabatic-to-diabatic transformation (ADT) angle [30] is also, up to a constant, identical to $\gamma(\mathbf{R})$ [31].] This GBO approach has been used to calculate the vibrational spectra of $\mathrm{H}_{3}$ [25] and $\mathrm{HD}_{2}$ [23,24].

The structure of this Letter is as follows. In Section 2, we survey the method, while the vibrational calculations for $\mathrm{D}_{3}, \mu \mathrm{H}_{2}, \mu \mathrm{HD}$, and $\mu \mathrm{DT}$ are reported in Section 3. The conclusions are in Section 4.

\section{Method}

For any isotopomer of a $\mathrm{X}_{3}$ system, the crossing seam in hyperspherical coordinates $(\rho, \theta, \varphi)$ is defined at an arbitrary value of the hyperradius $\rho$ by [31] 
$\varphi_{\mathrm{s}}=\tan ^{-1}\left[\frac{\cos \chi_{\mathrm{AC}}-t \cos \chi_{\mathrm{AB}}-\left(\frac{\mathrm{d}_{\mathrm{A}}}{\mathrm{d}_{\mathrm{C}}}\right)^{2}+t\left(\frac{\mathrm{d}_{\mathrm{A}}}{\mathrm{d}_{\mathrm{B}}}\right)^{2}}{\sin \chi_{\mathrm{AC}}+t \sin \chi_{\mathrm{AB}}}\right]$,

$\theta_{\mathrm{s}}=2 \sin ^{-1}\left[\frac{\left(\frac{\mathrm{d}_{\mathrm{A}}}{\mathrm{d}_{\mathrm{B}}}\right)^{2}-1}{\cos \left(\varphi_{\mathrm{s}}-\chi_{\mathrm{AB}}\right)-\left(\frac{\mathrm{d}_{\mathrm{A}}}{\mathrm{d}_{\mathrm{B}}}\right)^{2} \cos \varphi_{\mathrm{s}}}\right]$,

where

$t=\left[\left(\frac{\mathrm{d}_{\mathrm{A}}}{\mathrm{d}_{\mathrm{C}}}\right)^{2}-1\right]\left[\left(\frac{\mathrm{d}_{\mathrm{A}}}{\mathrm{d}_{\mathrm{B}}}\right)^{2}-1\right]^{-1}$

and

$d_{\mathrm{X}}^{2}=\frac{m_{\mathrm{X}}}{\mu}\left(1-\frac{m_{\mathrm{X}}}{M}\right)$

$\chi_{\mathrm{XY}}=2 \tan ^{-1}\left(\frac{m_{\mathrm{Z}}}{\mu}\right)$

$\mu=\sqrt{\frac{m_{\mathrm{A}} m_{\mathrm{B}} m_{\mathrm{C}}}{M}}$

$M=m_{\mathrm{A}}+m_{\mathrm{B}}+m_{\mathrm{C}}$

with $\mathrm{X}, \mathrm{Y}$, and $\mathrm{Z}$ standing for atoms $\mathrm{A}, \mathrm{B}$, and $\mathrm{C}$ with masses $m_{\mathrm{A}}, m_{\mathrm{B}}$, and $m_{\mathrm{C}}$. In case two atomic masses are equal, namely $m_{\mathrm{B}}=m_{\mathrm{C}}$, one gets the simplified expression

$\theta_{\mathrm{s}}=2 \sin ^{-1}\left|\frac{m_{\mathrm{B}}-m_{\mathrm{A}}}{m_{\mathrm{B}}+2 m_{\mathrm{A}}}\right|$,

while $\varphi_{\mathrm{s}}$ assumes the value $\pi(0)$ when $m_{\mathrm{A}}>m_{\mathrm{B}}$, and $0(\pi)$ when $m_{\mathrm{A}}<m_{\mathrm{B}}$; the value $\varphi_{\mathrm{s}}=0$ is chosen here for $m_{\mathrm{A}}<m_{\mathrm{B}}$. For example, for $\mathrm{HD}_{2}$, the seam is defined [31] by $\theta_{\mathrm{s}}=0.5048 \mathrm{rad}$ and $\varphi_{\mathrm{s}}=0$. In turn, for $\mu \mathrm{HD}$, one has $\theta_{\mathrm{s}}=1.8025 \mathrm{rad}$ and $\varphi_{\mathrm{s}}=0.1528 \mathrm{rad}$. Since $\theta_{\mathrm{s}} \neq 0$, only closed paths with $\theta \geqslant \theta_{\mathrm{s}}$ will therefore enclose the seam, with the accumulated phase change along the path being equal to $\pi$. For all other loops with $\theta<\theta_{\mathrm{s}}$, the accumulated phase change is 0 .

To calculate the vibrational levels, we solve the time-independent Schrödinger equation [25]

$\hat{H} \chi=E \chi$, where $\hat{H}=\hat{H}_{0}+\mathrm{i} \hat{H}_{1}$ is the system Hamiltonian,

$$
\begin{aligned}
\hat{H}_{0}= & -\frac{\hbar^{2}}{2 \mu}\left\{\frac{\partial^{2}}{\partial \rho^{2}}+\frac{16}{\rho^{2}}\right. \\
& \left.\times\left[\frac{1}{\sin \theta} \frac{\partial}{\partial \theta} \sin \theta \frac{\partial}{\partial \theta}+\frac{1}{4 \sin ^{2}(\theta / 2)} \frac{\partial^{2}}{\partial \phi^{2}}\right]\right\} \\
& +\frac{\hat{J}^{2}-\hat{J}_{z}^{2}}{\mu \rho^{2} \cos ^{2}(\theta / 2)}+\frac{\hat{J}_{z}^{2}+4 i \hbar \hat{J}_{z} \cos (\theta / 2)(\partial / \partial \phi)}{2 \mu \rho^{2} \sin ^{2}(\theta / 2)} \\
& +\frac{15 \hbar^{2}}{8 \mu \rho^{2}}+\frac{\sin (\theta / 2)}{\mu \rho^{2} \cos ^{2}(\theta / 2)} \frac{1}{2}\left[\hat{J}_{+}^{2}+\hat{J}_{-}^{2}\right] \\
& +V(\rho, \theta, \phi)+\frac{\hbar^{2}}{2 \mu}[\nabla \gamma(\rho, \theta, \phi)]^{2}
\end{aligned}
$$

and

$\hat{H}_{1}=\frac{\hbar^{2}}{2 \mu}\left[\nabla^{2} \gamma(\rho, \theta, \phi)+2 \nabla \gamma(\rho, \theta, \phi) \cdot \nabla\right]$,

where we have neglected all spin-orbit and spinspin interactions. Thus, $\hat{J}, \hat{J}_{z}$, and $\hat{J}_{ \pm}$are the total angular momentum, its component along the $z$ axis, and the raising/lowering operators in the body-fixed frame (respectively), which are defined by the set of external coordinates $(\alpha, \beta, \gamma)$; the external angle $\gamma$ should not be confused with the mixing angle $\gamma(\rho, \theta, \phi)$ which is determined by solving Eq. (7) of [31]. In turn, $V$ is the potential energy surface of the system, which depends only on the three internal coordinates $(\rho, \theta, \phi)$, with the hyperangles $\theta$ and $\phi$ being related [12] to the usual Smith-Whitten's $(\Theta, \Phi)$ hyperangles through the relations $\theta=\pi-4 \Theta$ and $\phi=2 \pi-$ $2 \Phi$. Thus, the range of $\theta$ extends from 0 to $\pi$, allowing to use Legendre (or Jacobi) polynomials in $\cos \theta$ as the finite basis representation (FBR) in $\theta$. Note that the GP effect may be turned-off by ignoring the imaginary operator $\mathrm{i} \hat{H}_{1}$ in the calculations. We emphasize that Eq. (8) to Eq. (9) should be strictly valid in the vicinity of the crossing seam, since the method [25] is based on the assumption that $\left(V_{2}-V_{1}\right) \chi_{2} \sim 0$ near the degeneracy locus where $V_{1}=V_{2} ; V_{i}$ and $\chi_{i}$ are, respectively, the adiabatic potential energy sheet and corresponding wave function for state $i$ $(=1,2)$. Although it was recognized [28] that 'certainly it is true that in a very small region, some features of the problem are insensitive to the 
difference in potential energies', such a methodology has been criticized on the basis that the most distinctive feature of the problem, which is the nature of the conical intersection, is missed'. It was further commented [28] that, in the near neighborhood of an intersection, one cannot assume $\chi_{2}$ to be very small'. The first criticism may have so many readings that we make no attempt of rebuttal. However, the second one seems unreasonable as we based our approach on the realistic assumption that $\chi_{2}$ should remain wellbehaved (in the sense of being a finite function) in the vicinity of the crossing seam. In fact, the behavior of the nuclear wave functions has been studied by Varandas and $\mathrm{Xu}$ [32] for the homonuclear isotopomers $\left(\mathrm{X}_{3}\right.$-type), who demonstrated that they vanish as a positive power of the radial coordinate $r$ as this approaches zero (i.e., in the vicinity of the conical intersection). Thus, the condition $\lim _{r \rightarrow 0}\left(V_{2}-V_{1}\right) \chi_{2}=0$ is satisfied for such systems, and most likely also for the heteronuclear isotopomers. To validate the method's general use, Varandas and $\mathrm{Xu}$ [25] have then simply recalled that the GP effect is a topological one known to be determined by the behavior of the potentials in the vicinity of the degeneracy $[6,7,17,29]$. Clearly, as it happens with any approximate theory (this is the case, since we have neglected the above coupling term), its validity can only be judged from the quality of the results to which it leads. In fact, a comparison of the cone states for $\mathrm{H}_{3}$ and $\mathrm{HD}_{2}$ calculated using the present approach with those obtained from more conventional methods has shown $[23,24]$ the results to be almost indistinguishable, especially for the low vibrational levels. On the other hand, the present method reveals itself to be rather more easy to implement computationally mostly due to the simplicity achieved by having introduced the mixing angle $\gamma(\rho, \theta, \phi)$. Thus, although the calculations presented below cannot be claimed to be exact, they should be accurate enough for seeking a criterion for the importance of the geometric phase effect in vibrational calculations for trihydrogen isotopomers. Stated differently, even if errors occur, there is no reason of principle to believe that such errors should affect distinctly the various systems considered in the present study.
To determine the action of the Hamiltonian $\hat{H}$ on the wave function (Lanczos vector), a mixed grid-basis method has been employed. Uniform grids have been utilized for the coordinates $\rho$ and $\varphi$, while the actions of the associated kinetic energy operators have been performed by using a prime-factor fast Fourier transform (PFFT) technique [12]. As in previous work [23-25], the calculations of the eigenenergies have been carried out for $J=0$ by using a minimum residual filter diagonalization (MFD) technique [33]; similarly to the simple Lanczos algorithm, the MFD approach has low storage requirements (only two iteration vectors) and utilizes just a singleLanczos recursion for the eigenvalue problem, while it can eliminate spurious and ghost eigenvalues.

\section{Results and discussion}

The calculations reported in the present work include the systems $\mathrm{D}_{3}, \mu \mathrm{H}_{2}, \mu \mathrm{HD}$, and $\mu \mathrm{DT}$, although numerical results will, for brevity, be reported only for $\mu \mathrm{H}_{2}$ and $\mu \mathrm{HD}$ (the numerical values for the other systems may be obtained from the authors upon request). Note that there has been a threefold reason for such a selection: (i) to have a further homonuclear system besides $\mathrm{H}_{3}$; (ii) to have a disparity of masses as large as possible such as to cover a wide range of $\theta_{\mathrm{s}}$ values; (iii) to accomodate systems without any permutational symmetry. All calculations employed the accurate $\mathrm{H}_{3}$ DMBE potential energy surface [34]. A grid of $N_{\rho} \times N_{\theta} \times N_{\varphi}=63 \times 70 \times 105$ has been employed covering the range $1.5 \leqslant \rho / \mathrm{a}_{0} \leqslant 11.5$. Fig. 1 illustrates the variation of the upper sheet of the potential energy surface along loops corresponding to an hyperradius of $\rho=6 \mathrm{a}_{0}$ and $\theta=\theta_{\mathrm{s}}$ (for the cases shown of $\mu \mathrm{H}_{2}$ and $\mu \mathrm{HD}$, the $\theta_{\mathrm{s}}$ values are 1.6192 and $1.8025 \mathrm{rad}$, respectively). For $\mu \mathrm{HD}$, a significant feature is perhaps the discontinuity at the conical intersection that arises at $\varphi=\varphi_{\mathrm{s}}$ $(0.1528 \mathrm{rad})$. In turn, Fig. 2 illustrates the variation of the geometric phase for two distinct values of $\theta$, one smaller and the other larger than $\theta_{\mathrm{s}}$. Clearly, for values $\theta<\theta_{\mathrm{s}}$, there is no sign change upon completion of a loop as this does not encircle 


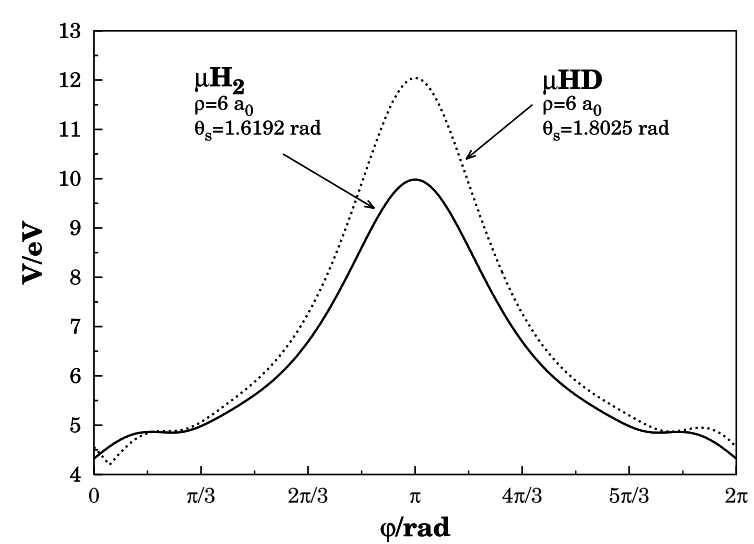

Fig. 1. Potential energy surface for the electronic-excited state as a function for $\varphi$ for fixed $\rho=6 \mathrm{a}_{0}$ and $\theta=\theta_{\mathrm{s}}: \mu \mathrm{H}_{2}$ (=1.6192 $\mathrm{rad})$, solid line; $\mu \mathrm{HD}(=1.8025 \mathrm{rad})$, dashed line.
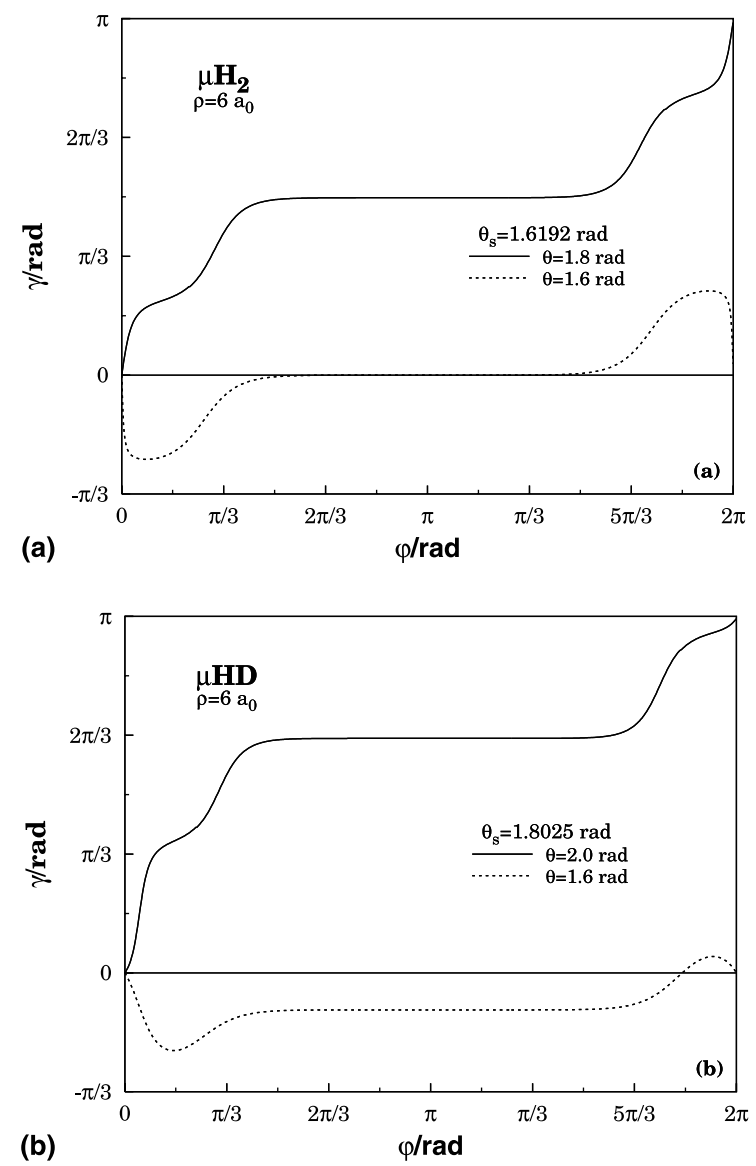

Fig. 2. Dependence of geometric phase on $\varphi$ and $\theta$ at $\rho=6 \mathrm{a}_{0}$ : (a) $\mu \mathrm{H}_{2}$; (b) $\mu \mathrm{HD}$. the conical intersection. Conversely, a sign change does occur for $\theta>\theta_{\mathrm{s}}$.

Table 1 compares the results obtained from two sets of calculations for $\mu \mathrm{H}_{2}$ and $\mu \mathrm{HD}$ covering energies up to about $E \sim 5 \mathrm{eV}$. All eigenvalues are quoted with four decimal figures, which are thought to be significant from the analysis of the associated error norms (these are typically $10^{-6} \mathrm{eV}$ ). The column headed NGP corresponds to the case where the GP effect is neglected, and conversely for the GP one which includes it. Clearly, most differences are smaller than the calculated error norms (and hence have been set equal to 0 for clarity). For example, for $\mu \mathrm{HD}$, except a few differences which are around $10^{-4} \mathrm{eV}$, in only one case it reaches $1.8 \times 10^{-3} \mathrm{eV}$. The results are compared with those obtained for $\mathrm{H}_{3}, \mathrm{D}_{3}$, $\mathrm{HD}_{2}$, and $\mu \mathrm{DT}$ in Fig. 3. It is seen that the differences between the NGP and GP results decrease along the sequence $\mathrm{H}_{3} \sim \mathrm{D}_{3}>\mathrm{HD}_{2}>\mu \mathrm{H}_{2}>$ $\mu \mathrm{HD}>\mu \mathrm{DT}$, as shown from the root-meansquare deviations for the lowest $M$ calculated levels:

$\Delta=\sqrt{\frac{\sum_{i=1}^{M}\left(\mathrm{GP}_{i}-\mathrm{NGP}_{i}\right)^{2}}{M}}$.

We pause to note that a more rigorous analysis should start with an assignment of the symmetry to the calculated vibrational levels. However, this would be a cumbersome task that would be virtually impossible to carry out accurately for the highly excited vibrational states of such floppy systems. On the other hand, for the ground state of the homonuclear trimers, we know $[16,17,25]$ to be comparing levels of $A_{1}$ (NGP) and $E$ (GP) symmetries. Thus, their energy difference is a genuine measure of the GP effect, which is exactly what we are aiming at. Using a bunch of $M$ levels may have the advantage of taking into account the GP effect on higher vibrational levels. Since we are aiming at such a phenomenological quantity to measure the GP effect on the spectra of the various isotopomers, the symmetry assignment was judged unjustified, and hence not done. Thus, $i$ in Eq. (11) indicates the level number irrespective of symmetry.

The logarithms of such deviations are displayed for $M=30$ in Fig. 4 as a function of the cosine of 
Table 1

Vibrational energy levels (in $\mathrm{eV}$ ) of $\mu \mathrm{H}_{2}$ and $\mu \mathrm{HD}$ calculated using the GBO approximation of [25]

\begin{tabular}{|c|c|c|c|c|c|c|}
\hline \multirow[t]{2}{*}{ Level } & \multicolumn{3}{|l|}{$\mu \mathrm{H}_{2}$} & \multicolumn{3}{|l|}{$\mu \mathrm{HD}$} \\
\hline & NGP & GP & $|\mathrm{NGP}-\mathrm{GP}|$ & NGP & GP & $|\mathrm{NGP}-\mathrm{GP}|$ \\
\hline 1 & 4.2271 & 4.2269 & $2 \times 10^{-4}$ & 4.1940 & 4.1940 & $0^{\mathrm{a}}$ \\
\hline 2 & 4.4402 & 4.4340 & $6.2 \times 10^{-3}$ & 4.3854 & 4.3854 & 0 \\
\hline 3 & 4.6212 & 4.6211 & $1 \times 10^{-4}$ & 4.5527 & 4.5527 & 0 \\
\hline 4 & 4.7655 & 4.7653 & $2 \times 10^{-4}$ & 4.6942 & 4.6942 & 0 \\
\hline 5 & 4.7956 & 4.7957 & $1 \times 10^{-4}$ & 4.7965 & 4.7965 & 0 \\
\hline 6 & 4.8049 & 4.8049 & 0 & 4.8021 & 4.8021 & 0 \\
\hline 7 & 4.8103 & 4.8103 & 0 & 4.8037 & 4.8037 & 0 \\
\hline 8 & 4.8197 & 4.8196 & $1 \times 10^{-4}$ & 4.8114 & 4.8114 & 0 \\
\hline 9 & 4.8284 & 4.8287 & $3 \times 10^{-4}$ & 4.8187 & 4.8187 & 0 \\
\hline 10 & 4.8348 & 4.8363 & $1.5 \times 10^{-3}$ & 4.8289 & 4.8290 & $1 \times 10^{-4}$ \\
\hline 11 & 4.8423 & 4.8426 & $3 \times 10^{-4}$ & 4.8332 & 4.8336 & $4 \times 10^{-4}$ \\
\hline 12 & 4.8465 & 4.8465 & 0 & 4.8430 & 4.8431 & $1 \times 10^{-4}$ \\
\hline 13 & 4.8525 & 4.8522 & $3 \times 10^{-4}$ & 4.8451 & 4.8452 & $1 \times 10^{-4}$ \\
\hline 14 & 4.8579 & 4.8580 & $1 \times 10^{-4}$ & 4.8467 & 4.8467 & 0 \\
\hline 15 & 4.8624 & 4.8624 & 0 & 4.8534 & 4.8534 & 0 \\
\hline 16 & 4.8640 & 4.8640 & 0 & 4.8569 & 4.8569 & 0 \\
\hline 17 & 4.8668 & 4.8671 & $3 \times 10^{-4}$ & 4.8607 & 4.8609 & $2 \times 10^{-4}$ \\
\hline 18 & 4.8687 & 4.8690 & $3 \times 10^{-4}$ & 4.8640 & 4.8640 & 0 \\
\hline 19 & 4.8717 & 4.8716 & $1 \times 10^{-4}$ & 4.8681 & 4.8681 & 0 \\
\hline 20 & 4.8734 & 4.8764 & $3 \times 10^{-4}$ & 4.8719 & 4.8725 & $6 \times 10^{-4}$ \\
\hline 21 & 4.8799 & 4.8799 & 0 & 4.8729 & 4.8747 & $1.8 \times 10^{-3}$ \\
\hline 22 & 4.8835 & 4.8862 & $2.7 \times 10^{-3}$ & 4.8772 & 4.8772 & 0 \\
\hline 23 & 4.8865 & 4.8873 & $8 \times 10^{-4}$ & 4.8799 & 4.8802 & $3 \times 10^{-4}$ \\
\hline 24 & 4.8915 & 4.8922 & $7 \times 10^{-4}$ & 4.8814 & 4.8814 & 0 \\
\hline 25 & 4.8942 & 4.8942 & 0 & 4.8877 & 4.8879 & $2 \times 10^{-4}$ \\
\hline 26 & 4.9017 & 4.8987 & $3 \times 10^{-4}$ & 4.8905 & 4.8904 & $1 \times 10^{-4}$ \\
\hline 27 & 4.9030 & 4.9043 & $1.3 \times 10^{-3}$ & 4.8926 & 4.8927 & $1 \times 10^{-4}$ \\
\hline 28 & 4.9036 & 4.9048 & $1.2 \times 10^{-3}$ & 4.8999 & 4.8999 & 0 \\
\hline 29 & 4.9100 & 4.9107 & $7 \times 10^{-4}$ & 4.9014 & 4.9015 & $1 \times 10^{-4}$ \\
\hline 30 & 4.9127 & 4.9126 & $1 \times 10^{-4}$ & 4.9023 & 4.9023 & 0 \\
\hline
\end{tabular}

${ }^{\text {a }}$ Smaller than the calculated error norm of $<10^{-6} \mathrm{eV}$, and hence defined as zero; see the text.

the tilting angle of the $\mathrm{C}_{3}$-axis, which increases from $\theta_{\mathrm{s}}=0$ in $\mathrm{H}_{3}$ up to $\theta_{\mathrm{s}}=2.2164 \mathrm{rad}$ in $\mu \mathrm{T}_{2}$ (see Table 2). In fact, the relative probability of looping $\left(\mathscr{P}_{\text {loop }}\right)$ around such an axis is expected to be some function of $\theta_{\mathrm{s}}$, which may be estimated by

$$
\begin{aligned}
\mathscr{P}_{\text {loop }} \propto & \int_{0}^{2 \pi} \int_{\theta_{\mathrm{s}}}^{\pi} \sin (\theta / 2) \mathrm{d} \theta \mathrm{d} \varphi / \int_{0}^{2 \pi} \int_{0}^{\pi} \\
& \times \sin (\theta / 2) \mathrm{d} \theta \mathrm{d} \varphi=\cos \left(\theta_{\mathrm{s}} / 2\right) .
\end{aligned}
$$

Fig. 4 shows that the results obtained for all systems studied thus far may be quantitavely described by the form

$\Delta=a \mathscr{P}_{\text {loop }}^{m}$

with the parameters (given with two decimal places) in the linear least-squares fitting procedure being $\ln (a / \mathrm{eV})=-2.37 \pm 1.29$ and $m=17.32 \pm$ 6.91. A similar result is obtained when using $M=20$ or 25 . Other two-parameter forms such as an inverse exponential in $\mathscr{P}_{\text {loop }}$ may also be viable, and hence the representation chosen in the present work should only be viewed as one amongst the simplest possible. Being not unique, the parameters in Eq. (13) cannot be claimed to be universal; numerical variations may then be expected, although the general trends should remain valid. Thus, rather than highlighting a particular functional form, we put the emphasis on the correlation observed between $\Delta$ and $\theta_{\mathrm{s}}$, which is shown to cover a wide range of values. It should also be noted that the reduction in the importance of the GP effect is already apparent when comparing the 


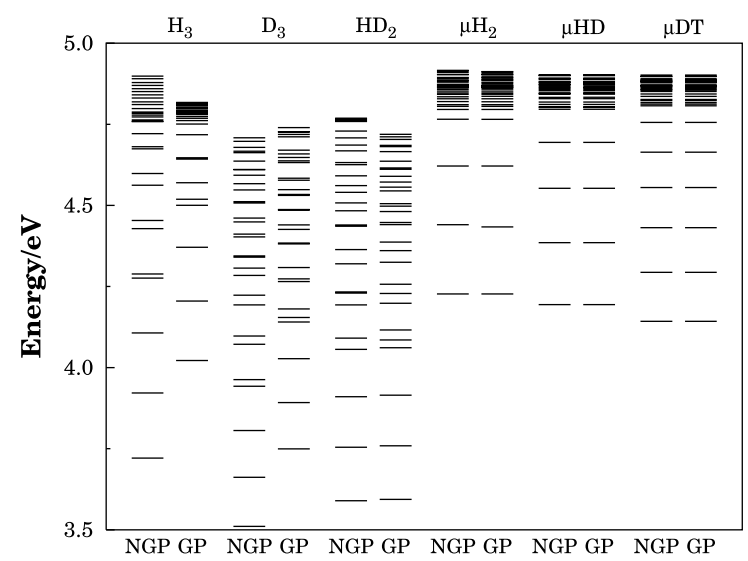

Fig. 3. A comparison of the lowest states $(E \leqslant 5 \mathrm{eV})$ of $\mathrm{H}_{3}$ [25], $\mathrm{D}_{3}, \mathrm{HD}_{2}$ [35, and references therein], $\mu \mathrm{H}_{2}, \mu \mathrm{HD}$, and $\mu \mathrm{DT}$, calculated without consideration (NGP) and with consideration (GP) of the geometric phase effect.

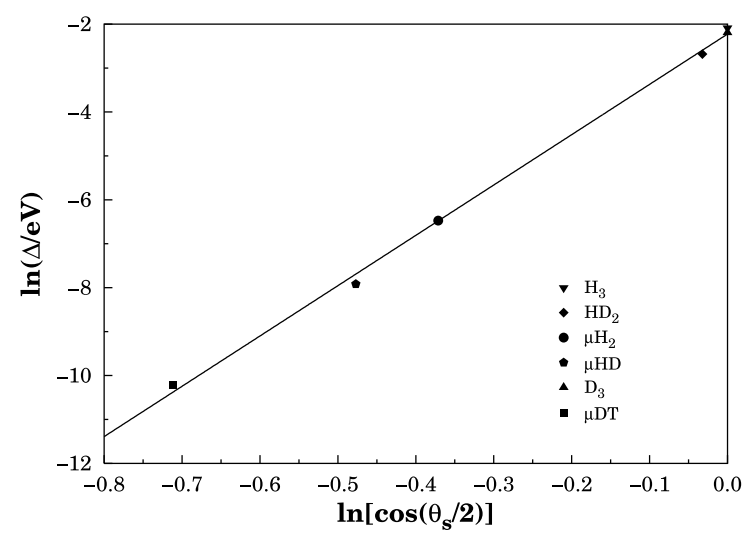

Fig. 4. Logarithm of the calculated root-mean-square deviation for the first 30 levels of $\mathrm{H}_{3}, \mathrm{D}_{3}, \mathrm{HD}_{2}, \mu \mathrm{H}_{2}, \mu \mathrm{HD}$, and $\mu \mathrm{DT}$ plotted as a function of $\mathscr{P}_{\text {loop }}\left(=\cos \theta_{\mathrm{s}} / 2\right)$, see Eq. (13).

energies for the vibrational ground state, i.e., without taking into consideration the averaging process in Eq. (11). In this case too, the small differences already observed for $\mathrm{HD}_{2}$ (and the heteronuclear isotopomers involving a muonium atom) may be attributed to the distinct atomic masses or, equivalently, to incomplete encircling of the locus of intersection.

Of course, the present criterion ignores the total energy, which is itself a very significant parameter when dealing with the dynamics of the exchange reactions in the lower sheet of the potential energy
Table 2

Values of $\theta_{\mathrm{s}}$ and $\varphi_{\mathrm{s}}$ for the various isotopomers of tri-hydrogen

\begin{tabular}{lll}
\hline System & $\begin{array}{l}\theta_{\mathrm{s}} \\
(\mathrm{rad})\end{array}$ & $\begin{array}{l}\varphi_{\mathrm{s}} \\
(\mathrm{rad})\end{array}$ \\
\hline$\mu_{3}$ & 0 & 0 \\
$\mu_{2} \mathrm{H}$ & 0.8668 & $\pi$ \\
$\mu_{2} \mathrm{D}$ & 0.9534 & $\pi$ \\
$\mu_{2} \mathrm{~T}$ & 0.9837 & $\pi$ \\
$\mu \mathrm{H}_{2}$ & 1.6192 & 0 \\
$\mu \mathrm{HD}$ & 1.8025 & 0.1528 \\
$\mu \mathrm{HT}$ & 1.8743 & 0.2133 \\
$\mu \mathrm{D}_{2}$ & 2.0241 & 0 \\
$\mu \mathrm{DT}$ & 2.1150 & 0.0650 \\
$\mu \mathrm{T}_{2}$ & 2.2164 & 0 \\
$\mathrm{H}_{3}$ & 0 & 0 \\
$\mathrm{H}_{2} \mathrm{D}$ & 0.4023 & $\pi$ \\
$\mathrm{H}_{2} \mathrm{~T}$ & 0.5786 & $\pi$ \\
$\mathrm{HD}_{2}$ & 0.5048 & 0 \\
$\mathrm{HDT}$ & 0.6667 & 0.3386 \\
$\mathrm{HT}_{2}$ & 0.8211 & 0 \\
$\mathrm{D}_{3}$ & 0 & 0 \\
$\mathrm{D}_{2} \mathrm{~T}$ & 0.2497 & $\pi$ \\
$\mathrm{DT}_{2}$ & 0.2854 & 0 \\
$\mathrm{~T}_{3}$ & 0 & 0 \\
\hline
\end{tabular}

surface (as already observed, the energy must be at least high enough to allow a loop around the conical intersection). However, its role may not be so decisive when studying the vibrational spectroscopy of the upper cone, since it is always larger than the energy of the crossing seam. Despite such a limitation, the simple correlation in Eq. (13) is gratifying, and may be used to estimate the role of the GP effect in vibrational calculations of the cone states for other isotopomers not studied thus far. Thus, the present results suggest that, although significant for the vibrational states of homonuclear tri-hydrogenic systems, the GP effect is significantly less so for their isotopomers in particular those involving a single-muonium particle. We emphasize that this result should not drastically depend on the form of the geometric phase. In fact, it has been noted [31] that physical quantities such as vibrational levels should be gauge invariant with respect to different functional forms adopted for the angle around the conical intersection.

Although vibrational states may have little to do with reactive scattering, they both stem from the same potential energy surface and hence are influenced (possibly in different ways) by mani- 
festations of the same geometric phase effect. Thus, we conclude as we have began by addressing the case of an elementary reaction such as $\mathrm{A}+\mathrm{BC} \rightarrow \mathrm{AB}+\mathrm{C}$. We conjecture that the impact of the GP effect may in this case get further diminished since, in a classical sense, it is not mandatory for a reactive trajectory that it completes an entire loop around the crossing seam before yielding the products. In fact, the probability of doing so should increase with impact parameter, and hence with the value of $J$, as it really appears to be the case $[10,11]$ for $H+\mathrm{D}_{2}$.

\section{Conclusions}

A single-surface Born-Oppenheimer equation recently proposed [25] has been used to calculate the cone states of $\mathrm{D}_{3}, \mu \mathrm{H}_{2}, \mu \mathrm{HD}$, and $\mu \mathrm{DT}$. In all cases involving muonion atoms, we observe significantly smaller differences between the NGP and GP eigenvalues than for $\mathrm{H}_{3}, \mathrm{D}_{3}$ or even $\mathrm{HD}_{2}$. Such a result is rationalized with basis on the value of $\theta_{\mathrm{s}}$, the tilting angle of the $\mathrm{C}_{3}$-axis in $\mathrm{H}_{3}$ due to mass effects. A simple criterion involving configuration space arguments has therefore been proposed and shown empirically to describe well the average deviations between the NGP and GP results. Clearly, the title issue deserves further investigation.

\section{Acknowledgements}

This work has been supported by the Fundação para a Ciência e Tecnologia, Portugal.

\section{References}

[1] Y.-S.M. Wu, A. Kuppermann, Chem. Phys. Lett. 235 (1995) 105

[2] L. Schnieder, K. Seekamp-Rahn, E. Wrede, K.W. Welge, J. Chem. Phys. 107 (1997) 6175.

[3] S.A. Harich, D. Dai, X. Yang, S.D. Chao, R.T. Skodje, J. Chem. Phys. 116 (2002) 4769.

[4] A. Varandas, H. Yu, J. Mol. Struct. Theochem. 81 (1999) 4933.
[5] C.A. Mead, D.G. Truhlar, J. Chem. Phys. 70 (1979) 2284.

[6] G. Herzberg, H.C. Longuet-Higgins, Faraday Discuss. Chem. Soc. 35 (1963) 77.

[7] H.C. Longuet-Higgins, Proc. R. Soc. Lond. A 344 (1975) 147.

[8] M.V. Berry, Proc. R. Soc. A 392 (1984) 45.

[9] T.N. Kitsopoulos, M.A. Buntine, D.P. Balwin, R.N. Zare, D.W. Chandler, Science 260 (1993) 1605

[10] B.K. Kendrick, J. Chem. Phys. 114 (2001) 4335.

[11] A. Kuppermann, Y.-S.M. Wu, Is the geometric phase effect important for the $\mathrm{H}+\mathrm{D}_{2} \rightarrow \mathrm{HD}+\mathrm{D}$ reaction? Workshop on Quantum Reaction Dynamics, Pasadena, USA, 2001.

[12] A.J.C. Varandas, H.G. Yu, J. Chem. Soc. Faraday Trans. 93 (1997) 819.

[13] B.K. Kendrick, Phys. Rev. Lett. 79 (1997) 2431.

[14] S. Mahapatra, H. Köppel, J. Chem. Phys. 109 (1998) 1721.

[15] H. Busch, V. Dev, H.-A. Eckel, S. Kasahara, J. Wang, W. Demtröder, P. Sebald, W. Meyer, Phys. Rev. Lett. 81 (1998) 4584.

[16] A.J.C. Varandas, Z.R. Xu, Int. J. Quantum Chem. 75 (1999) 89.

[17] A.J.C. Varandas, Z.R. Xu, in: M. Baer, G.D. Billing (Eds.), Adv. Chem. Phys., vol. 124, Wiley, New York, 2001, p. 659.

[18] A.J.C. Varandas, J. Tennyson, J.N. Murrell, Chem. Phys. Lett. 61 (1979) 431.

[19] A. Kuppermann, Y.-S.M. Wu, Chem. Phys. Lett. 213 (1993) 636.

[20] C.A. Mead, J. Chem. Phys. 72 (1980) 3839.

[21] G.D. Billing, N. Marković, J. Chem. Phys. 99 (1993) 2674.

[22] A. Kuppermann, Y.-S.M. Wu, Chem. Phys. Lett. 205 (1993) 577.

[23] Z.R. Xu, A.J.C. Varandas, Int. J. Quantum Chem. 80 (2000) 454.

[24] Z.R. Xu, A.J.C. Varandas, J. Phys. Chem. 105 (2001) 2246.

[25] A.J.C. Varandas, Z.R. Xu, J. Chem. Phys. 112 (2000) 2121.

[26] M. Baer, R. Englman, Chem. Phys. Lett. 265 (1997) 105.

[27] M. Baer, J. Chem. Phys. 107 (1997) 2694.

[28] B. Kendrick, C.A. Mead, D.G. Truhlar, Chem. Phys. Lett. 277 (2002) 31

[29] M.S. Child, H.C. Longuet-Higgins, Philos. Trans. R. Soc. Lond. A 254 (1961) 259.

[30] M. Baer, Chem. Phys. Lett. 35 (1975) 112.

[31] Z.R. Xu, M. Baer, A.J.C. Varandas, J. Chem. Phys. 112 (2000) 2746.

[32] A.J.C. Varandas, Z.R. Xu, Chem. Phys. Lett. 316 (2000) 248.

[33] H.G. Yu, S.C. Smith, Ber. Bunsenges. Phys. Chem. 101 (1997) 400.

[34] A.J.C. Varandas, F.B. Brown, C.A. Mead, D.G. Truhlar, N.C. Blais, J. Chem. Phys. 86 (1987) 6258.

[35] Z.R. Xu, A.J.C. Varandas, Int. J. Quantum Chem. 83 (2001) 279. 